\title{
Generation of a Bio-intensive Strategy using Chitosan Formulations and Ampelomyces quisqualis for the Management of Powdery Mildew of Grapes
}

\author{
Ratna U. Thosar ${ }^{1 *}$, Indu Sawant ${ }^{1}$, Vijayshree M. Chavan ${ }^{1}$, Sanjay D. Sawant ${ }^{2}$, \\ Sujoy Saha $^{1}$ and A. Vikram Suthakar ${ }^{3}$ \\ ${ }^{1}$ Department of Plant Pathology, ICAR-National Research Centre for Grapes, \\ Pune - 412307, Maharashtra, India \\ ${ }^{2}$ BalasahebSawantKonkanKrishiVidyapeeth, Dapoli-415712, Maharashtra, India \\ ${ }^{3}$ AuraphyllInnoventures India Pvt. Limited, Coimbatore- 641035, TamilNadu, India \\ *Corresponding author
}

\section{A B S T R A C T}

\begin{tabular}{|l|}
\hline Key w or d s \\
Chitosan, Powdery \\
mildew, Grapes, \\
$\begin{array}{l}\text { Erysiphe necator, } \\
\text { Ampelomycesquisqu } \\
\text { alis }\end{array}$ \\
\hline Article Info \\
\hline $\begin{array}{l}\text { Accepted: } \\
\text { 10 September } 2020 \\
\text { Available Online: } \\
\text { 10 October } 2020\end{array}$ \\
\hline
\end{tabular}

\section{Keywords}

Chitosan, Powdery mildew, Grapes, Ampelomycesquisqu alis

Article Info

Accepted: 10 September 2020 Available Online

Powdery mildew caused by Erysiphe necator is one of the most important diseases of grapevine in India. It affects the grape production by causing significant reduction in yield and quality of grapes. Chitosan is a deactivated derivative of chitin and the efficacy of three chitosan formulations, Chitosan fulvate @ 2ml/L, Chitosan oligosaccharides @ 2ml/L, Chitosan@4ml/L were evaluated against powdery mildew. The physiological loss in weight of berries was also assessed. During the fruiting seasons of 2018-2019 and 20192020, chitosan formulations were evaluated both as solo treatments and in alternation with Ampelomyces quisqualis @ 5ml/L. All Chitosan formulations effectively inhibited powdery mildew on leaves and berries when compared to untreated control. Among solo treatments, chitosan exhibited lower PDI (17.76 and 5.29) on leaves than untreated control (24.28 and 13.12) during 2018-2019 and 2019-2020 respectively. In case of bunches similar trend was observed with PDI 23.39 and 9.90 against untreated control 30.31 and 17.18 in both seasons. However, the formulations were found more effective when used in alternation with Ampelomyces quisqualis. During 2018-2019 and 2019-2020, Chitosan / Ampelomyces quisqualis treatment recorded significantly lower PDI of powdery mildew on leaves i.e.12.55 and 4.77than the untreated control with PDI 24.28 and 13.22 respectively. In case of bunches, treatment Chitosan / Ampelomyces quisqualis showed the lowest disease severity with PDI 19.28 and 8.98 against untreated control with PDI 31.60 and 17.18 in both the seasons respectively. The applications of formulation containing chitosan alternated with sprays of Ampelomyces quisqualis $(5 \mathrm{ml} / \mathrm{L})$ showed least disease as well physiological loss in weight of grapes. All chitosan formulations were effective in minimizing physiological loss in weight of grapes as compared to control. Among all treatments, Chitosan / Ampelomyces quisqualis treatment recorded the lowest PLW i.e.13.94 and 13.90, whereas untreated control showed the highest PLW i. e. 15.85 and 15.87 during both seasons respectively. Results showed that chitosan can be effectively used in powdery mildew management, either alone or in alternation with the bioformulation of Ampelomyces quisqualis. 


\section{Introduction}

Grape is an important commercial fruit crop and is most widely cultivated in temperate, sub-tropical and tropical regions of the world. This crop occupies fifth position amongst fruit crops in India with a production of 1.21 million tonnes. The area under grape cultivation in India is 137 thousand hectares with production of 2951 thousand MT (Anonymous, 2019). The commercial grape varieties are susceptible to several diseases. Among these, the powdery mildew caused due to Erysiphe necator Schwein. (syn. Uncinula necator Schwein.), is one of the most severe diseases in all grape growing areas (Iriti et al., 2011, Sharma et al., 2017).

The malady is characterized by white powder like growth on the leaves, immature shoots and young berries and is responsible for affecting the quality and yield of grapes worldwide (Gadoury et al., 2012). Fungicides are the major tools in the control of the disease [23] but non-rational use of fungicides leads to health risk, both to the environment and human being. The congruent effect of the rising environmental risk due to xenobiotics along with the selection of pathogen strains resistant to chemicals and the cost of traditional pesticide treatments have prompted the studies on alternative strategies in crop protection. Moreover, in the recent times, a lot of emphasis is given on minimum pesticide residues in grapes, especially in export grapes to the European Union and the search for alternatives became imperative. Alternative or low profile compounds like ozonated water (Fujiwara and Fujii, 2002) chlorine dioxide (Sharma et al., 2017) etc. are used in the post veraison stage to control powdery mildew disease of grapes.

Chitosan ( $\beta$-1, 4-D-glucosamine polymer) is a natural, safe, and cheap biopolymer produced from chitin, the major constituent of arthropods exoskeleton and fungal cell walls and the second renewable carbon source after lignocellulosic biomass (Barber et al 1989). Chitosan oligosaccharide is a mixture of oligomers of D-glucosamine. The plants' response to chitin, chitosan, and derived oligosaccharides depends on the acetylation degree of these compounds which indicates possible biocontrol regulation of plant immune system (Akiyama et al., 1995, CordLandwehr et al., 2016, Li et al., 2016) and the degree of polymerization (Walker-Simmons and Ryan 1984, Li et al., 2016) of these compounds. Chitosan and its oligomers have been used for a plethora of reasons in crop husbandry viz. as biopesticides, biofertilisers, elicitor for induction of natural resistance of plant, seed coating formulations and agricultural film (Ha and Huang 2007, El Hadrami et al., 2010; Trouvelot et al., 2014) due to its unique attributes such as biodegradability, nontoxicity, biocompatibility and fungicidal effect (Rinaudo, 2006, Kumar, 2000). Chitosan treatment increases plants tolerance to the attack of wide range of soil and foliar pathogens and induces root nodulation as well (Hamel et al., 2010).

Keeping this at the backdrop, the efficacy of chitosan and oligo-chitosan formulations were assessed against grapevine powdery mildew disease.

\section{Materials and Methods}

The efficacy of three chitosan formulations provided by the AuraphyllInnoventures India Pvt. Limited viz. Chitosan fulvate (Chit'o'sun) @ 2ml/L, Chitosan oligosaccharides (Chitang) @ 2ml/L and Chitosan (Vilasae) @ 4ml/L were evaluated against powdery mildew infection on grape leaves and berries of cv. Manik Chaman. The trial was conducted at an experimental vineyard of ICAR-National Research Centre 
for Grapes, Pune (latitude 18.31N, longitude 73.55 E)during fruiting season 2018-2019 and 2019-2020.

The trial was set up as a Randomised block design with four replications, with 10 vines (an experimental plot) per treatment in each block. Chitosan formulations were evaluated both as solo treatments and in alternation with
Ampelomycesquisqualis@ 5ml/L. A total of 8 treatments were taken into account. Applications were given at 7 days interval from the beginning of grape susceptibility to powdery mildew until the completion of veraison. Treatment with sprays of Ampelomyces quisqualis @ $5 \mathrm{ml} / \mathrm{L}$ and untreated control were used as check.

\begin{tabular}{|c|l|c|c|}
\hline $\begin{array}{c}\text { Sr. } \\
\text { No. }\end{array}$ & \multicolumn{1}{|c|}{ Treatments } & Dose & $\begin{array}{c}\text { Total no. of } \\
\text { applications }\end{array}$ \\
\hline $\mathbf{1}$ & Chitosan fulvate & $2 \mathrm{ml}$ & 5 \\
\hline $\mathbf{2}$ & Chitosan oligosaccharides & $2 \mathrm{ml}$ & 5 \\
\hline $\mathbf{3}$ & Chitosan & $4 \mathrm{ml}$ & 5 \\
\hline $\mathbf{4}$ & Ampelomyces quisqualis & $5 \mathrm{ml}$ & 5 \\
\hline $\mathbf{5}$ & Chitosan fulvate / Ampelomyces quisqualis & $2 \mathrm{ml} / 5 \mathrm{ml}$ & $3 / 2$ \\
\hline $\mathbf{6}$ & Chitosan oligosaccharides /Ampelomyces quisqualis & $2 \mathrm{ml} / 5 \mathrm{ml}$ & $3 / 2$ \\
\hline $\mathbf{7}$ & Chitosan /Ampelomyces quisqualis & $4 \mathrm{ml} / 5 \mathrm{ml}$ & $3 / 2$ \\
\hline $\mathbf{8}$ & Untreated Control & -- & -- \\
\hline
\end{tabular}

To avoid spray drift to neighbouring plots, sprayings were carried out with knapsack sprayers. Symptoms were assessed weekly on leaves and bunches, by visual inspections and disease severity were evaluated on a scale of $0-5$, where $0=$ no symptoms; $1=1-10 \% ; 2$ $=11-25 \% ; 3=26-50 \% ; 4=51-75 \%$; and $5=$ $76-100 \%$ of infected leaf areas or infected berries per bunch, respectively (Horsfall and Heuberger, 1942). Data regarding disease severity were processed according to McKinney, 1923, in order to calculate the percentage of disease severity (PDI):

$\mathrm{PDI}=\frac{\text { sum of numerical ratings }}{\text { No. of leaves } / \text { bunches observed }} \times \frac{100}{\text { Maximum rating }}$

The physiological loss in weight of berries was also assessed. Weight of bunches were recorded for all treatments at 24 hour intervals for first 5-7 days at room temperature. The percent loss of weight over initial weight was calculated mathematically for grapes in each replicate.

\section{Statistical analysis}

The data were analyzed in RBD design with analysis of variance (ANOVA) using SAS (ver. 9.3; SAS Institute Inc., Cary, North Carolina, USA). The percentage data was arcsine-transformed before analysis.

\section{Results and Discussion}

\section{Bio-efficacy of chitosan formulations on grapes leaves}

In 2018-2019 and 2019-2020, first disease observations on leaves in experimental plot were recorded on $15^{\text {th }}$ January 2019 and $02^{\text {nd }}$ January 2020 respectively (Table 1). The disease severity increased slowly from first to last observation. The PDI of powdery mildew on the leaves were gradually increased from 22.34 to 24.28 and 11.29 to 13.12 in the unsprayed control respectively. Chitosan formulations effectively inhibited powdery mildew on leaves when compared to untreated control. However, the formulations 
were found more effective when used in alternation with Ampelomyces quisqualis (Table 1). In the last observation recorded on 12.2.19, Chitosan / Ampelomyces quisqualis treatment recorded significantly lower PDI of powdery mildew on leaves i.e.12.55than the untreated control (24.28). The alternation of chitosan fulvate, chitosan oligosaccharides and Chitosan with Ampelomyces quisqualis were statistically at par with each other with PDI of 14.22, 13.82 and 12.55 respectively. In case of solo treatment, chitosan exhibited lower PDI (17.76) than other treatments but all the solo treatments were statistically on par with each other.

In 2019-2020, similar trend was observed as previous trial. All treatments performed significantly superior over untreated control. Chitosan /Ampelomyces quisqualis showed lower PDI (4.77) than other treatments during last observation. All the treatments except untreated control were statistically at par with each other. As the disease incidence was negligible on leaves during 2019-20, less variation in the result of different treatments were observed. The percent disease intensity on leaves in untreated control plot was 13.12.

In case of bunches, similar trend was observed (Table 2). The last observation recorded on 12.2.2019 showed that treatment Chitosan/Ampelomyces quisqualis recorded a significantly lower PDI of powdery mildew on bunches i.e.19.28 than the untreated control (31.60). All the treatments were significantly superior over untreated control. Treatments Chitosan/Ampelomyces quisqualis and Chitosan oligosaccharides/Ampelomyces quisqualis were at par with each other and significantly superior over other treatments. Treatment Ampelomyces quisqualis and Chitosan fulvate / Ampelomycesquisqualis were at par with each other with a PDI values of 20.99 and 21.39 respectively.
In 2019-2020, the last observation recorded on 28.01.2020 showed that treatment Chitosan/Ampelomyces quisqualis recorded a significantly lower PDI of powdery mildew on bunches i.e. 8.98 than the untreated control (17.18). All the treatments were significantly superior over untreated control. Chitosan/Ampelomyces quisqualis, Chitosan oligosaccharides/Ampelomyces quisqualis, Chitosan fulvate / Ampelomyces quisqualis and Chitosan treatments were at par with each other. Treatment Ampelomyces quisqualis and Chitosan oligosaccharides were at par with each other.

\section{Physiological loss in weight (PLW)}

Results (Table 3) showed the significant differences among the treatments during the storage at ambient conditions. Control recorded higher PLW than all the treatments. By increasing the storage duration, the PLW also increased.

In 2018-2019, on third day of storage, physiological loss in weight (PLW) in control reached up to 13.73, whereas PLW in Chitosan / Ampelomyces quisqualis was significantly lesser(11.77) than untreated control. Chitosan treatment and Chitosan oligosaccharides / Ampelomyces quisqualis treatment registered with values of less than 5 per cent. On $4^{\text {th }}$ day, PLW values were more than $5 \%$ in all treatments, but the untreated control had the highest value of 15.87. PLW value in Chitosan / Ampelomyces quisqualis treatment was only 13.94. Similar observations were also recorded in season 2019-2020. On third day of storage, physiological loss in weight (PLW) in control reached up to 14.04, whereas PLW in Chitosan / Ampelomyces quisqualis recorded significantly lesser (11.73) than untreated control. Remaining treatments also registered with values of less than 5 per cent. 
Table.1 Effect of different chitosan formulations on powdery mildew severity in grapes leaves during 2018-2019 and 2019-2020

\begin{tabular}{|c|c|c|c|c|c|c|c|c|c|c|c|}
\hline \multirow{2}{*}{$\begin{array}{l}\text { Tr. } \\
\text { No. }\end{array}$} & \multirow[t]{2}{*}{ Tr. Name } & \multicolumn{5}{|c|}{ PDI (2018-2019) } & \multicolumn{5}{|c|}{ PDI (2019-2020) } \\
\hline & & $15 / 01 / 19$ & 22/01/19 & 29/01/19 & $05 / 02 / 19$ & $12 / 02 / 19$ & $02 / 01 / 20$ & $09 / 01 / 20$ & $16 / 01 / 20$ & $23 / 01 / 20$ & $30 / 01 / 20$ \\
\hline $\mathbf{1}$ & Chitosan fulvate & $\begin{array}{c}0.00 \\
(0.00) \mathrm{a}\end{array}$ & $\begin{array}{c}10.10(18.39) \\
\mathrm{d}\end{array}$ & $\begin{array}{c}11.20 \\
(19.53) \mathrm{b}\end{array}$ & $\begin{array}{c}11.30 \\
(19.62) b\end{array}$ & $\begin{array}{c}11.40 \\
(19.71) \mathrm{b}\end{array}$ & $\begin{array}{c}0.00 \\
(0.00) \mathrm{a}\end{array}$ & $\begin{array}{c}0.35 \\
(3.38) \mathrm{a}\end{array}$ & $\begin{array}{c}0.4 \\
(3.55) \mathrm{a}\end{array}$ & $\begin{array}{c}0.65 \\
(4.59) \mathrm{a}\end{array}$ & $\begin{array}{c}0.95 \\
(5.58) \mathrm{a}\end{array}$ \\
\hline 2 & Chitosan oligosaccharides & $\begin{array}{c}0.00 \\
(0.00) \mathrm{a}\end{array}$ & $\begin{array}{c}8.70(17.15) \\
\mathrm{cd}\end{array}$ & $\begin{array}{c}10.35 \\
(18.61) b\end{array}$ & $\begin{array}{c}10.50 \\
(18.76) b\end{array}$ & $\begin{array}{c}10.60 \\
(18.86) b\end{array}$ & $\begin{array}{c}0.00 \\
(0.00) \mathrm{a}\end{array}$ & $\begin{array}{c}0.35 \\
(3.38) \mathrm{a}\end{array}$ & $\begin{array}{c}0.5 \\
(4.04) \mathrm{a}\end{array}$ & $\begin{array}{c}0.75 \\
(4.95) \mathrm{a}\end{array}$ & $\begin{array}{c}0.95 \\
(5.58) \mathrm{a}\end{array}$ \\
\hline 3 & Chitosan & $\begin{array}{c}0.00 \\
(0.00) \mathrm{a}\end{array}$ & $\begin{array}{c}6.70(14.94) \\
\text { bc }\end{array}$ & $\begin{array}{c}9.00(17.46) \\
b\end{array}$ & $\begin{array}{c}9.10 \\
(17.56) \mathrm{b}\end{array}$ & $\begin{array}{c}9.30(17.76) \\
b\end{array}$ & $\begin{array}{c}0.00 \\
(0.00) \mathrm{a}\end{array}$ & $\begin{array}{c}0.20 \\
(1.81) \mathrm{a}\end{array}$ & $\begin{array}{c}0.55 \\
(4.23) \mathrm{a}\end{array}$ & $\begin{array}{c}0.55 \\
(4.20) \mathrm{a}\end{array}$ & $\begin{array}{c}0.85 \\
(5.29) \mathrm{a}\end{array}$ \\
\hline 4 & Ampelomyces quisqualis & $\begin{array}{c}0.00 \\
(0.00) \mathrm{a}\end{array}$ & $\begin{array}{c}5.45(13.40) \\
\mathrm{ab}\end{array}$ & $\begin{array}{c}5.80 \\
(13.86) a\end{array}$ & $\begin{array}{c}5.90 \\
(14.00) \mathrm{a}\end{array}$ & $\begin{array}{c}6.15(14.32) \\
a\end{array}$ & $\begin{array}{c}0.00 \\
(0.00) \mathrm{a}\end{array}$ & $\begin{array}{c}0.25 \\
(2.01) \mathrm{a}\end{array}$ & $\begin{array}{c}0.65 \\
(4.61) \mathrm{a}\end{array}$ & $\begin{array}{c}0.70 \\
(4.22) \mathrm{a}\end{array}$ & $\begin{array}{c}0.85 \\
(5.29) \mathrm{a}\end{array}$ \\
\hline 5 & $\begin{array}{l}\text { Chitosan fulvate / } \\
\text { Ampelomycesquisqualis }\end{array}$ & $\begin{array}{c}0.00 \\
(0.00) \mathrm{a}\end{array}$ & $\begin{array}{c}5.30(13.28) \\
\mathrm{ab}\end{array}$ & $\begin{array}{c}5.75 \\
(13.85) \mathrm{a}\end{array}$ & $\begin{array}{c}5.85 \\
(13.97) \mathrm{a}\end{array}$ & $\begin{array}{c}6.05(14.22) \\
\mathrm{a}\end{array}$ & $\begin{array}{c}0.00 \\
(0.00) \mathrm{a}\end{array}$ & $\begin{array}{c}0.30 \\
(2.72) \mathrm{a}\end{array}$ & $\begin{array}{c}0.50 \\
(4.04) \mathrm{a}\end{array}$ & $\begin{array}{c}0.70 \\
(4.22) \mathrm{a}\end{array}$ & $\begin{array}{c}0.9 \\
(5.44) \mathrm{a}\end{array}$ \\
\hline 6 & $\begin{array}{l}\text { Chitosan oligosaccharides } \\
\text { / Ampelomyces quisqualis }\end{array}$ & $\begin{array}{c}0.00 \\
(0.00) \mathrm{a}\end{array}$ & $\begin{array}{c}4.75(13.55) \\
\mathrm{ab}\end{array}$ & $\begin{array}{c}5.40 \\
(13.37) \mathrm{a}\end{array}$ & $\begin{array}{c}5.55 \\
(13.56) \mathrm{a}\end{array}$ & $\begin{array}{c}5.75(13.82) \\
a\end{array}$ & $\begin{array}{c}0.00 \\
(0.00) \mathrm{a}\end{array}$ & $\begin{array}{c}0.15 \\
(1.55) \mathrm{a}\end{array}$ & $\begin{array}{c}0.40 \\
(3.55) \mathrm{a}\end{array}$ & $\begin{array}{c}0.45 \\
(3.84) \mathrm{a}\end{array}$ & $\begin{array}{c}0.75 \\
(4.96) \mathrm{a}\end{array}$ \\
\hline 7 & $\begin{array}{l}\text { Chitosan /Ampelomyces } \\
\text { quisqualis }\end{array}$ & $\begin{array}{c}0.00 \\
(0.00) \mathrm{a}\end{array}$ & $\begin{array}{c}3.90(11.90) \\
\mathrm{a}\end{array}$ & $\begin{array}{c}4.35 \\
(11.93) \mathrm{a}\end{array}$ & $\begin{array}{c}4.60 \\
(12.28) \mathrm{a}\end{array}$ & $\begin{array}{c}4.80(12.55) \\
\mathrm{a}\end{array}$ & $\begin{array}{c}0.00 \\
(0.00) \mathrm{a}\end{array}$ & $\begin{array}{c}0.35 \\
(3.36) a\end{array}$ & $\begin{array}{c}0.55 \\
(4.23) \mathrm{a}\end{array}$ & $\begin{array}{c}0.65 \\
(4.59) \mathrm{a}\end{array}$ & $\begin{array}{c}0.70 \\
(4.77) \mathrm{a}\end{array}$ \\
\hline 8 & Untreated Control & $\begin{array}{c}10.60 \\
(18.88) b\end{array}$ & $\begin{array}{c}14.60 \\
(22.34) \mathrm{e}\end{array}$ & $\begin{array}{c}16.70 \\
(23.90) c\end{array}$ & $\begin{array}{c}16.90 \\
(24.08) c\end{array}$ & $\begin{array}{c}17.15 \\
(24.28) c\end{array}$ & $\begin{array}{c}2.15(8.35) \\
b\end{array}$ & $\begin{array}{c}3.85 \\
(11.29) b\end{array}$ & $\begin{array}{c}4.2 \\
(11.76) b\end{array}$ & $\begin{array}{c}4.75 \\
(12.58) \mathrm{b}\end{array}$ & $\begin{array}{c}5.20 \\
(13.12) b\end{array}$ \\
\hline & LSD $(p=0.05)$ & 1.38 & 2.62 & 3.24 & 3.05 & 2.96 & 0.71 & 2.19 & 1.10 & 1.68 & 0.87 \\
\hline
\end{tabular}

Means with the same letter are not significantly different. Fig. in the parenthesis show angular transform values 
Table.2 Effect of different chitosan formulations on powdery mildew severity on grapes bunches

\begin{tabular}{|c|c|c|c|c|c|c|c|c|c|c|c|}
\hline \multirow{2}{*}{$\begin{array}{l}\text { Tr. } \\
\text { No. }\end{array}$} & \multirow[t]{2}{*}{ Tr. Name } & \multicolumn{5}{|c|}{ PDI (2018-2019) } & \multicolumn{5}{|c|}{ PDI (2019-2020) } \\
\hline & & $15 / 01 / 19$ & 22/01/19 & 29/01/19 & $05 / 02 / 19$ & $12 / 02 / 19$ & $02 / 01 / 20$ & 08/01/20 & $15 / 01 / 20$ & $22 / 01 / 20$ & $28 / 01 / 20$ \\
\hline $\mathbf{1}$ & Chitosan fulvate & $\begin{array}{c}0.00 \\
(0.00) \mathrm{a}\end{array}$ & $\begin{array}{c}10.50 \\
(18.82) \mathrm{a}\end{array}$ & $\begin{array}{c}16.00 \\
(23.48) b\end{array}$ & $\begin{array}{c}17.50 \\
(24.62) \mathrm{c}\end{array}$ & $\begin{array}{c}19.50 \\
(26.12) \mathrm{c}\end{array}$ & $\begin{array}{c}0.00 \\
(0.00) \mathrm{a}\end{array}$ & $\begin{array}{c}2.50 \\
(9.06) \mathrm{de}\end{array}$ & $\begin{array}{c}3.00 \\
(3.54) \mathrm{a}\end{array}$ & $\begin{array}{c}4.00 \\
(11.42) \mathrm{b}\end{array}$ & $\begin{array}{c}5.50 \\
(13.51) b\end{array}$ \\
\hline 2 & Chitosan oligosaccharides & $\begin{array}{c}0.00 \\
(0.00) \mathrm{a}\end{array}$ & $\begin{array}{c}12.50 \\
(20.52) \mathrm{ab}\end{array}$ & $\begin{array}{c}15.50 \\
(23.10) \mathrm{b}\end{array}$ & $\begin{array}{c}16.50 \\
(23.83) b c\end{array}$ & $\begin{array}{c}18.50 \\
(25.36) b c\end{array}$ & $\begin{array}{c}0.00 \\
(0.00) \mathrm{a}\end{array}$ & $\begin{array}{c}2.00 \\
(8.13) \mathrm{dc}\end{array}$ & $\begin{array}{c}2.25 \\
(4.02) \mathrm{a}\end{array}$ & $\begin{array}{c}2.75 \\
(9.44) \mathrm{ab}\end{array}$ & $\begin{array}{c}4.25 \\
(11.83) \mathrm{ab}\end{array}$ \\
\hline 3 & Chitosan & $\begin{array}{c}0.00 \\
(0.00) \mathrm{a}\end{array}$ & $\begin{array}{c}15.50 \\
(22.99) b\end{array}$ & $\begin{array}{c}13.50 \\
(21.44) \mathrm{ab}\end{array}$ & $\begin{array}{c}14.00 \\
(21.74) \mathrm{abc}\end{array}$ & $\begin{array}{c}16.00 \\
(23.39) \mathrm{abc}\end{array}$ & $\begin{array}{c}0.00 \\
(0.00) \mathrm{a}\end{array}$ & $\begin{array}{c}1.50 \\
(6.93) \mathrm{bc}\end{array}$ & $\begin{array}{c}1.75 \\
(4.23) \mathrm{a}\end{array}$ & $\begin{array}{c}2.75 \\
(9.44) \mathrm{ab}\end{array}$ & $\begin{array}{c}3.00 \\
(9.90) \mathrm{a}\end{array}$ \\
\hline 4 & Ampelomyces quisqualis & $\begin{array}{c}0.00 \\
(0.00) \mathrm{a}\end{array}$ & $\begin{array}{c}8.50 \\
(16.93) \mathrm{a}\end{array}$ & $\begin{array}{c}10.00 \\
(18.39) \mathrm{a}\end{array}$ & $\begin{array}{c}11.00 \\
(19.17) \mathrm{ab}\end{array}$ & $\begin{array}{c}13.00 \\
(20.99) \mathrm{ab}\end{array}$ & $\begin{array}{c}0.00 \\
(0.00) \mathrm{a}\end{array}$ & $\begin{array}{l}2.25 \\
(8.62) \text { edc }\end{array}$ & $\begin{array}{c}2.50 \\
(4.61) \mathrm{a}\end{array}$ & $\begin{array}{c}2.50 \\
(9.05) \mathrm{ab}\end{array}$ & $\begin{array}{c}3.50 \\
(10.64) \mathrm{ab}\end{array}$ \\
\hline 5 & $\begin{array}{l}\text { Chitosan fulvate / } \\
\text { Ampelomyces quisqualis }\end{array}$ & $\begin{array}{c}0.00 \\
(0.00) \mathrm{a}\end{array}$ & $\begin{array}{c}10.00 \\
(18.21) \mathrm{a}\end{array}$ & $\begin{array}{c}12.00 \\
(20.10) \mathrm{ab}\end{array}$ & $\begin{array}{c}12.50 \\
(20.56) a b c\end{array}$ & $\begin{array}{c}13.50 \\
(21.39) \mathrm{ab}\end{array}$ & $\begin{array}{c}0.00 \\
(0.00) \mathrm{a}\end{array}$ & $\begin{array}{c}1.50 \\
(6.97) \mathrm{bc}\end{array}$ & $\begin{array}{c}1.50 \\
(4.04) \mathrm{a}\end{array}$ & $\begin{array}{c}2.25 \\
(8.43) \mathrm{ab}\end{array}$ & $\begin{array}{c}3.25 \\
(9.65) \mathrm{a}\end{array}$ \\
\hline 6 & $\begin{array}{l}\text { Chitosan oligosaccharides } \\
\text { /Ampelomyces quisqualis }\end{array}$ & $\begin{array}{c}0.00 \\
(0.00) \mathrm{a}\end{array}$ & $\begin{array}{r}9.00 \\
(17.43) \mathrm{a}\end{array}$ & $\begin{array}{c}10.00 \\
(18.05) \mathrm{a}\end{array}$ & $\begin{array}{c}10.50 \\
(18.71) \mathrm{a}\end{array}$ & $\begin{array}{c}12.00 \\
(20.14) \mathrm{a}\end{array}$ & $\begin{array}{c}0.00 \\
(0.00) \mathrm{a}\end{array}$ & $\begin{array}{c}1.25 \\
(6.21) \mathrm{b}\end{array}$ & $\begin{array}{c}1.75 \\
(3.54) \mathrm{a}\end{array}$ & $\begin{array}{c}2.00 \\
(7.79) \mathrm{a}\end{array}$ & $\begin{array}{c}2.75 \\
(9.07) \mathrm{a}\end{array}$ \\
\hline 7 & $\begin{array}{l}\text { Chitosan /Ampelomyces } \\
\text { quisqualis }\end{array}$ & $\begin{array}{c}0.00 \\
(0.00) \mathrm{a}\end{array}$ & $\begin{array}{c}8.50 \\
(16.67) \mathrm{a}\end{array}$ & $\begin{array}{c}9.50 \\
(17.89) \mathrm{a}\end{array}$ & $\begin{array}{c}10.00 \\
(18.35) \mathrm{a}\end{array}$ & $\begin{array}{c}11.00 \\
(19.28) \mathrm{a}\end{array}$ & $\begin{array}{c}0.00 \\
(0.00) \mathrm{a}\end{array}$ & $\begin{array}{c}0.40 \\
(3.61) \mathrm{a}\end{array}$ & $\begin{array}{c}1.25 \\
(4.23) \mathrm{a}\end{array}$ & $\begin{array}{c}1.75 \\
(7.29) \mathrm{a}\end{array}$ & $\begin{array}{c}2.50 \\
(8.98) \mathrm{a}\end{array}$ \\
\hline 8 & Untreated Control & $\begin{array}{c}12.50 \\
(20.60) b\end{array}$ & $\begin{array}{c}24.50 \\
(29.66) \mathrm{c}\end{array}$ & $\begin{array}{c}25.00 \\
(29.96) \mathrm{c}\end{array}$ & $\begin{array}{c}25.50 \\
(30.31) \mathrm{d}\end{array}$ & $\begin{array}{c}27.50 \\
(31.60) \mathrm{d}\end{array}$ & $\begin{array}{c}3.00 \\
(9.79) \mathrm{b}\end{array}$ & $\begin{array}{c}3.25 \\
(10.29) \mathrm{e}\end{array}$ & $\begin{array}{c}5.25 \\
(11.76) \mathrm{b}\end{array}$ & $\begin{array}{c}7.5 \\
(15.67) \mathrm{c}\end{array}$ & $\begin{array}{r}8.75 \\
(17.18) \mathrm{c}\end{array}$ \\
\hline & $\operatorname{LSD}(p=0.05)$ & 1.36 & 4.16 & 4.29 & 4.66 & 4.49 & 1.21 & 1.83 & 1.10 & 3.13 & 3.08 \\
\hline
\end{tabular}

Means with the same letter are not significantly different. Fig. in the parenthesis show angular transform values 
Table.3 Effect of application of different chitosan formulations on physiological loss in weight of grapes

\begin{tabular}{|c|c|c|c|c|c|c|c|c|c|}
\hline \multirow[t]{2}{*}{ Tr. No. } & \multirow[t]{2}{*}{ Tr. Name } & \multicolumn{4}{|c|}{ 2018-2019 } & \multicolumn{4}{|c|}{ 2019-2020 } \\
\hline & & $1^{\text {st day }}$ & $2^{\text {nd }}$ day & $3^{\text {rd }}$ day & $4^{\text {th }}$ day & $1^{\text {st }}$ day & $2^{\text {nd }}$ day & $3^{\text {rd }}$ day & $4^{\text {th }}$ day \\
\hline 1 & Chitosan fulvate & $\begin{array}{c}1.50 \\
(7.00) \mathrm{ab}\end{array}$ & $\begin{array}{c}3.59 \\
(10.90) \mathrm{cd}\end{array}$ & $\begin{array}{c}5.07 \\
(13.01) \mathrm{bcd}\end{array}$ & $\begin{array}{c}7.14 \\
(15.49) \text { cde }\end{array}$ & $\begin{array}{c}2.26 \\
(8.63) \mathrm{a}\end{array}$ & $\begin{array}{c}3.62 \\
(10.97) a b\end{array}$ & $\begin{array}{c}4.36 \\
(11.75) \mathrm{a}\end{array}$ & $\begin{array}{c}6.22 \\
(14.42) a b\end{array}$ \\
\hline 2 & Chitosan oligosaccharides & $\begin{array}{c}1.73 \\
(7.56) \mathrm{cd}\end{array}$ & $\begin{array}{c}3.21 \\
(10.32) b c\end{array}$ & $\begin{array}{c}5.28 \\
(13.27) \mathrm{cd}\end{array}$ & $\begin{array}{c}6.59 \\
(14.86) \mathrm{abcd}\end{array}$ & $\begin{array}{c}2.32 \\
(8.74) \mathrm{ab}\end{array}$ & $\begin{array}{c}3.52 \\
(10.81) \mathrm{ab}\end{array}$ & $\begin{array}{c}4.46 \\
(12.16) \mathrm{a}\end{array}$ & $\begin{array}{c}5.93 \\
(13.99) \mathrm{a}\end{array}$ \\
\hline 3 & Chitosan & $\begin{array}{c}1.33 \\
(6.61) \mathrm{a}\end{array}$ & $\begin{array}{c}2.98 \\
(9.93) \mathrm{b}\end{array}$ & $\begin{array}{c}4.72 \\
(12.54) \mathrm{abc}\end{array}$ & $\begin{array}{c}6.37 \\
(14.61) \mathrm{ab}\end{array}$ & $\begin{array}{c}2.26 \\
(8.63) \mathrm{a}\end{array}$ & $\begin{array}{c}3.47 \\
(10.72) \mathrm{ab}\end{array}$ & $\begin{array}{c}4.27 \\
(11.91) \mathrm{a}\end{array}$ & $\begin{array}{c}5.92 \\
(14.08) \mathrm{a}\end{array}$ \\
\hline 4 & Ampelomyces quisqualis & $\begin{array}{c}1.76 \\
(7.61) \mathrm{d}\end{array}$ & $\begin{array}{c}3.63 \\
(10.98) \mathrm{cd}\end{array}$ & $\begin{array}{c}5.64 \\
(13.72) d\end{array}$ & $\begin{array}{c}7.34 \\
(15.79) \mathrm{de}\end{array}$ & $\begin{array}{c}2.48 \\
(9.00) \mathrm{ab}\end{array}$ & $\begin{array}{c}3.72 \\
(11.08) \mathrm{ab}\end{array}$ & $\begin{array}{c}4.78 \\
(12.62) \mathrm{ab}\end{array}$ & $\begin{array}{c}6.24 \\
(14.45) \mathrm{ab}\end{array}$ \\
\hline 5 & $\begin{array}{l}\text { Chitosan fulvate / Ampelomyces } \\
\text { quisqualis }\end{array}$ & $\begin{array}{l}1.74 \\
(7.58) \mathrm{cd}\end{array}$ & $\begin{array}{c}3.46 \\
(10.69) \mathrm{cd}\end{array}$ & $\begin{array}{c}5.19 \\
(13.15) \mathrm{bcd}\end{array}$ & $\begin{array}{c}6.88 \\
(15.19) \text { bcde }\end{array}$ & $\begin{array}{c}2.41 \\
(8.93) \mathrm{ab}\end{array}$ & $\begin{array}{c}3.59 \\
(10.49) \mathrm{a}\end{array}$ & $\begin{array}{r}4.40 \\
(12.11) \mathrm{a}\end{array}$ & $\begin{array}{c}6.11 \\
(14.30) \mathrm{a}\end{array}$ \\
\hline 6 & $\begin{array}{l}\text { Chitosan oligosaccharides } \\
\text { /Ampelomyces quisqualis }\end{array}$ & $\begin{array}{r}1.32 \\
(6.56) \mathrm{a}\end{array}$ & $\begin{array}{c}2.89 \\
(9.76) \mathrm{b}\end{array}$ & $\begin{array}{c}4.63 \\
(12.40) \mathrm{ab}\end{array}$ & $\begin{array}{c}6.32 \\
(14.54) \mathrm{ab}\end{array}$ & $\begin{array}{c}2.29 \\
(8.69) \mathrm{bc}\end{array}$ & $\begin{array}{c}3.31 \\
(10.45) \mathrm{a}\end{array}$ & $\begin{array}{r}4.24 \\
(11.87) \mathrm{a}\end{array}$ & $\begin{array}{c}5.85 \\
(13.91) \mathrm{a}\end{array}$ \\
\hline 7 & Chitosan /Ampelomyces quisqualis & $\begin{array}{c}1.50 \\
(7.01) \mathrm{abc}\end{array}$ & $\begin{array}{c}2.54 \\
(9.14) \mathrm{a}\end{array}$ & $\begin{array}{c}4.18 \\
(11.77) \mathrm{a}\end{array}$ & $\begin{array}{c}5.83 \\
(13.94) \mathrm{a}\end{array}$ & $\begin{array}{c}2.07 \\
(8.23) \mathrm{a}\end{array}$ & $\begin{array}{c}2.93 \\
(8.81) \mathrm{a}\end{array}$ & $\begin{array}{c}4.15 \\
(11.73) \mathrm{a}\end{array}$ & $\begin{array}{c}5.80 \\
(13.90) \mathrm{a}\end{array}$ \\
\hline 8 & Untreated Control & $\begin{array}{c}1.84 \\
(7.78) d\end{array}$ & $\begin{array}{c}3.66 \\
(11.01) \mathrm{d}\end{array}$ & $\begin{array}{c}5.65 \\
(13.73) d\end{array}$ & $\begin{array}{c}7.50 \\
(15.87) \text { e }\end{array}$ & $\begin{array}{c}3.31 \\
(10.45) \mathrm{c}\end{array}$ & $\begin{array}{c}4.66 \\
(12.47) \mathrm{b}\end{array}$ & $\begin{array}{c}5.89 \\
(14.04) b\end{array}$ & $\begin{array}{c}7.46 \\
(15.85) b\end{array}$ \\
\hline & $\operatorname{LSD}(\mathrm{p}=0.05)$ & 0.57 & 0.68 & 0.71 & 0.92 & 1.10 & 1.84 & 1.63 & 1.47 \\
\hline
\end{tabular}

Means with the same letter are not significantly different. Fig. in the parenthesis show angular transform values 
On $4^{\text {th }}$ day, PLW values were more than $5 \%$ in all treatments, but the untreated control had the highest value of 15.85. PLW value in Chitosan / Ampelomyces quisqualis treatment was only 13.90. Chitosan / Ampelomyces quisqualis treatment registered better results among all treatments.

In conventionally managed vineyards, powdery mildew is usually controlled by regular application of fungicides. However, the health and environmental impact associated with the use of these products, the development of resistance to certain fungicides and the demand for residue-free grapes provide incentives for minimising reliance on chemicals in viticulture, pointing to a need for effective organic alternatives (Gubler et al., 1996, Hofstein et al., 1996, Savocchia et al., 2004). Chitosan as a foliar treatment is a good option to control the growth, spread and development of many diseases involving viruses, bacteria, fungi and pests (Rabea et al., 2003). It is nontoxic for humans and also has a low environmental impact (Li et al., 1992, Shahidi et al., 1999). Chitosan oligomers of different molecular weight and degree of acetylation induced an accumulation of phytoalexins in grapevine leaves, which reduced $B$. cinerea and Plasmopara viticola infections (Ben-Shalom and Fallik, 2003). The induction of systemic resistance in plants with natural compounds like chitosan, is a promising approach to disease control (Gozzo, 2003). Kamble et al., (2019) reported that preventive sprays of GI (Gamma irradiated) chitosan reduced the powdery mildew intensity in pea.

In the present study, three chitosan formulations, solo and in alternation with Ampelomyces quisqualis were evaluated for their field efficacy in the control of powdery mildew of grapes. Results revealed that chitosan formulations effectively inhibited powdery mildew on grapes when compared to untreated control. Results of the present investigations on the efficacy of chitosan formulations had close resemblance with earlier investigations. Iriti et al., (2011) evaluated the new chitosan formulation and reported the significant reduction in the PDI of powdery mildew particularly at the concentration of $0.1 \%$, even under high disease pressure conditions. Chitosan provided the best grapevine downy mildew protection without affecting the quality of grape production (Romanazzi et al., 2016). Earlier investigations recorded the efficacy of chitosan against powdery mildew (Blumeria graminis f. sp.hordei) in barley, as well as its effective antiviral activity in tobacco and bean crops (Iriti et al., 2006, Faoro et al., 2008, Iriti and Faoro 2008).

Combined application of BCAs with different plant elicitors to target different stages in the infection process, was one of the approaches to increase the reliability and level of activity of biological control treatments (Schmitt et al., 2001). Where a single biological method does not give sufficient protection, such approach is essential (Elad et al., 1996). In our studies, the formulations were found more effective when used in alternation with Ampelomyces quisqualis. Results of the present investigations are in tandem with earlier investigations indicating the effectiveness of chitosan with bio-control agents. Giotiset al., (2012) used the combination of biological control agents (BCAs) Ampelomyces quisqualis, plant defence elicitors Milsana® VP 2002 (a plant extract of Reynoutria sachalinensis) and chitosan and reported the significant reduction in cucumber powdery mildew incidence as compared to untreated control. El-Mohamedy et al., (2014) analysed T. harzianum and chitosan (0.5 and $1.0 \mathrm{~g} / \mathrm{l})$ as combined treatments for controlling Fusarium crown and root rot of tomato and reported that the combination had significantly reduced 
Fusarium crown and root rot disease. The genus Ampelomyces is a potential biocontrol agent against the powdery mildew pathogens (Kanipriya et al., 2019) and Kiss (2003) reported its commercial formulation in controlling the powdery mildew menace.

Biocides are effective in maintaining the physiological loss in weight (PLW) of grapes. Chlorine dioxide $\left(\mathrm{ClO}_{2}\right)$ played an important role in reducing the PLW at $200 \mathrm{ppm}$ concentration (Sharma et al., 2017). In our study the physiological loss in weight (PLW) was low in all treated plots as compared to untreated control plot. Treatment chitosan /Ampelomyces quisqualis showed significantly lower physiological loss in weight (PLW) than untreated control. Chitosan coating improves the shelf life and postharvest quality of table grape (Suryawanshi, 2018). It is clear that, chitosan application played an important role in reducing the PLW. Hence, chitosan can be effectively used in powdery mildew management as well as improve the shelf life of berries, either alone or in alternation with Ampelomyces quisqualis.

Our study revealed that chitosan formulations have a potential to control the plant diseases. Though interesting theoretical and applied findings were gathered in recent years, more are needed to examine the mechanisms governing the mode of action of these compounds. In the case of antimicrobial mode of action, future work should aim at clarifying the molecular details of the underlying mechanisms and their relevance to the antimicrobial activity of chitosan. Therefore, future research should be directed towards understanding their molecular level details, which may provide insights into the unknown biochemical functions of chitosan formulations as well as help to accelerate their future and might assist in the goal of sustainable agriculture.

\section{Acknowledgements}

The authors are thankful to the Director, ICAR- National Research Centre for Grapes, Pune for taking initiative and providing the facilities. Also thanks to Auraphyll Innoventures India Pvt Ltd, Erode for supplying the samples required for the experiment.

\section{References}

Anonymous (2019). http://agricoop.nic.in/ sites/default/files/2018-

$19 \% 20 \% 281$ st $\% 20 \quad$ Adv.Est_.\%

29_updt. Pdf.

Akiyama, K., K. Kawazu, Kobayashi, A. (1995). Partially N-deacetylated chitin oligomers (pentamer to heptamer) are potential elicitors for (+)-pisatin induction in pea epicotyls. Z. Naturforsch. 50:391- 397.

Barber, M., R. E. Bertram, Ride, J. (1989). Chitin oligosaccharides elicit lignification in wounded wheat leaves. Physiological and Molecular Plant Pathology, 34:3-12.

Ben-Shalom, Noah andFallik, E. (2003). Further suppression of Botrytis cinerea disease in cucumber seedlings by chitosan-copper complex as compared with chitosan alone. Phytoparasitica. 31:99-102. 10.1007/BF02979773.

Cord-Landwehr, S., Melcher, R.L.J., Kolkenbrock, S., Moerschbacher B. M. (2016). A chitin deacetylase from the endophytic fungus Pestalotiopsis $s p$. efficiently inactivates the elicitor activity of chitin oligomers in rice cells. Sci Rep. 6.

El Hadrami, A, Adam, L.R., El Hadrami, I., Daayf, F. (2010). Chitosan in plant protection. Mar Drugs. 8:968-987.

El-Mohamedy, R.S.R., Abdel-Kareem, F., Jabnoun-Khiareddine, $\mathrm{H}$ and 
DaamiRemadi, M. (2014). Chitosan and Trichoderma harzianum as fungicide alternatives for controlling Fusarium crown and root rot of tomato. Tunis. J. Plant Prot 9: 31-43.

Elad,Y.,Malathrakis, N.E., Dik, A.J. (1996). Biologica1 contro1 of Botrytis-incited diseases and powdery mildews in greenhouse crops. Crop Prot 15:229240.

Faoro, F. Maffi, D., Cantu, D. \&Iriti, M. (2008). Chemical-induced resistance against powdery mildew in barley: The effects of chitosan and benzothiadiazole. BioControl. 53:387401. 10.1007/s10526-007-9091-3.

Fujiwara, K. andFujii, T. (2002). Effects of spraying Ozonated water on the severity of powdery mildew infection on cucumber leaves. OZONE-SCI ENG, 24(6):463-469. doi:10.1080/01919510208901635.

Gadoury, D., Cadle-davidson, L., Wilcox, W., Dry, I., Seem, R. and Milgroom, M. (2012). Grapevine powdery mildew (Erysiphe necator): a fascinating system for the study of the biology, ecology and epidemiology of an obligate biotroph. Mol. Plant Pathol 13(1):1-16.

Giotis, C., Markellou, E., Theodoropoulou, A., Kostoulas, G., Wilcockson, S. and Leifert, C. (2012). The effects of different biological control agents (BCAs) and plant defence elicitors on cucumber powdery mildew (Podosphaera xanthii). Org. Agr. 2:89-101.

Gozzo, F. (2003). Systemic acquired resistance in crop protection: from nature to a chemical approach. J. Agric. Food Chem. 51, 4487-4503.

Gubler, W.D., Ypema, H. L., Ouimette, D.G. and Bettiga, L.J. (1996). Occurrence of resistance in Uncinula necator to triadimefon, myclobutanil and fenarimol in California grapevine. Plant Dis 80:902-909.

Ha, M. T. and Huang, J. W. (2007). Control of Fusarium wilt of asparagus bean by organic soil amendment and microorganisms. Plant Pathol Bull. 16:169-180.

Hamel, L.P. and Beaudoin, N. (2010). Chitooligosaccharide sensing and downstream signaling: Contrasted outcomes in pathogenic and beneficial plant-microbe interactions. Planta 232:787-806.

Hofstein, R., Daoust, R. A. and Aeschlimann, J. P. (1996). Constraints to the development of biofungicides: the example of 'AQ10', a new product for controlling powdery mildews. Entomophaga41:455-460.

Horsfall, J. G., Heuberger, J. W. (1942). Measuring magnitude of a defoliation disease in tomatoes. Phytopathol 32:226-232.

Iriti, M., Sironi, M., Gomarasca, S., Casazza,A. P., Soave, C. and Faoro, F. (2006). Cell death-mediated antiviral effect of chitosan in tobacco. Plant Physiol. Biochem44(11-12): 893-900.

Iriti, M. and Faoro, F. (2008).Abscisic acid is involved in chitosan-induced resistance to tobacco necrosis virus (TNV) Plant Physiol. Biochem. 46(12):1106-1111.

Iriti, M., Vitalini, S., Ditommaso, G., D'amico, S., Borgo, M. and Faoro, F. (2011). New chitosan formulation prevents grapevine powdery mildew infection and improves polyphenol content and free radical scavenging activity of grape and wine. Aust. J. Grape Wine R.17:263-269.

Kamble, P. K., Lohate, S.R., Hasabnis, S.N., Dalvi, S.G. and Chaudhari, S.R. (2019). Efficacy of GI Chitosan in Management of Powdery Mildew of Pea. Int. J.Curr. Microbiol. App.Sci. 
8(12): 1879-1885.

Kanipriya, R., Rajendran, L., Raguchander, T. and Karthikeyan, G. (2019). Characterization of Ampelomyces and its potentiality as an effective biocontrol agent against Erysipheci choracearum DC causing powdery mildew disease in Bhendi (Abelmoschus esculentus (L.) Moench). Madras Agric. J. 106, SpI :267-278.

Kiss, L. (2003). A review of fungal antagonists of powdery mildews and their potential as biocontrol agents. Pest Man. Sci., 59: 475-483.

Kumar , M. N. V. R. (2000). A review of chitin and chitosan applications. React. Funct. Polym. 46: 1-27.

Li, P., Linhardt, R. J., Cao, Z. 2016. Structural characterization of oligochitosan elicitor from Fusarium sambucinum and its elicitation of defensive responses in Zanthoxylum bungeanum. Int. J. Mol. Sci. 17:12.

Li, Q., Dunn, E.T., Grandmaison, E.W., Goosen, M.F.A., 1992. Applications and properties of chitosan. J. Bioact. Compat. Polym. 7:370-397.

McKinney, H. H. (1923). Influence of soil temperature and moisture on infection of wheat seedlings by Helminthosporium sativum. J. Agri. Res., 26:195-217.

Rabea, E.I., El Badawy, M.T., Stevens, C.V., Smagghe, G., Steurbaut, W. (2003). Chitosan as antimicrobial agent: Applications and mode of action. Biomacromolecules, 4: 1457-1465.

Rebollar-Alviter, A. and Mizuho, N. (2011). Optimizing Fungicide Applications for Plant Disease Management: Case Studies on Strawberry and Grape. 10.5772/26740.

Rinaudo, M. (2006). Chitin and Chitosan: Properties and applications. Prog. Polym. Sci., 31: 603-632.
Romanazzi, G., Mancini, V., Feliziani, E., Servili, A., Endeshaw, S., and Neri, D. (2016). Impact of alternative fungicides on grape downy mildew control and vine growth and development. Plant Dis. 100:1-10.

Schmitt, A., Malathrakis, N. E., Konstantinidou-Doltsinis, S., Dik, A., Ernst, A., Francke, W., PetsikosPanayotarou, N., Schuld, M., Seddon, B. (2001). Improved plant health by the combination of biological disease control methods. Bulletin OILB/ SROP. International Organization for Biological and Integrated Control of Noxious Animals and Plants (OIBC/OILB), West Palaearctic Regional Section (WPRS/SROP), Dijon, France 24(3):29-32.

Savocchia, S., Stummer, B., Wicks, T., Heeswijck, R. and Scott, E. (2004). Reduced sensitivity of Uncinula necator to sterol demethylation inhibiting fungicides in southern Australian vineyards, Australia. Plant Pathol. $33: 465-473$ 10.1071/AP04066.

Shahidi, F., Arachchi, J. K.V., Jeon, Y. J.(1999). Food applications of chitin and chitosan. Trends Food Sci. Technol. 10: 37-51.

Sharma, A.K., Sawant, I S.,Sawant, S.D.,Saha,S., Kadam P. and Somkuwar R.G. (2017). Aqueous chlorine dioxide for the management of powdery mildew vis-a-vis maintaining quality of grapes and raisins. Journal of Eco-friendly Agriculture12(2): 59-64.

Shiri, M. A., Bakhshi, D., Ghasemnezhad, M., Dadi M., Papachatzis, A., Kalorizou, H. (2013). Chitosan coating improves the shelf life and postharvest quality of table grape (Vitis vinifera) cultivar Shahroudi. Turk J Agric For, 148-156. Suryawanshi, K. T. (2018). Development of 
disease management strategies for minimal fungicides residue in table grapes. Ph.D. Thesis, Indira Gandhi KrishiVishwavidyalaya Raipur (Chhattisgarh).

Trouvelot, S., Héloir, M.C., Poinssot, B., Gauthier, A., Paris, F., Guillier, C., Combier, M., Trdá, L., Daire, X., Adrian, M. (2014). Carbohydrates in plant immunity and plant protection: roles and potential application as foliar sprays. Front Plant Sci. 5:592.

Walker-Simmons, M., Ryan, C. A. (1984). Proteinase inhibitor synthesis in tomato leaves: induction by chitosan oligomers and chemically modified chitosan and chitin. Plant Physiol. 76(3): 787-790.

\section{How to cite this article:}

Ratna U. Thosar, Indu Sawant, Vijayshree M. Chavan, Sanjay D. Sawant, Sujoy Saha and Vikram Suthakar, A. 2020. Generation of a Bio-intensive Strategy using Chitosan Formulations and Ampelomyces quisqualis for the Management of Powdery Mildew of Grapes. Int.J.Curr.Microbiol.App.Sci. 9(10): 1190-1201. doi: https://doi.org/10.20546/ijcmas.2020.910.143 